\title{
An Upper Bound Solution for Continued Compression of a Cylinder
}

\author{
Sergei ALEXANDROV, Dmitry LISOVENKO, Marko VILOTIC
}

\begin{abstract}
A sequential upper bound method for the cylindrical compression test is developed. The method uses rigid and plastic regions to build up a kinematically admissible velocity field. A technique for calculating the power dissipation in continued compression is described. A numerical method is only necessary to evaluate ordinary integrals. An illustrative example presented shows that some important parameters of the process are not sensitive to the friction factor if its value is high enough. This observation is in qualitative agreement with a finite element solution.
\end{abstract}

Keywords: continued compression; friction; rigid region; singularity; upper bound method

\section{INTRODUCTION}

The cylindrical compression test is often used for studying the evolution of various material properties [1-4] and frictional conditions for metal forming applications [58]. A widely accepted method of theoretical analysis of this test is based on the upper bound theorem. In general, upper bound solutions can be conveniently divided into two categories. Instantaneous solutions fall into one of these categories. These solutions are only valid at the initial instant. Therefore, the profile of specimens after finite deformation cannot be predicted. Typical solutions of this category for analysis of the cylindrical compression test are proposed in $[5,9,10]$. A barrel parameter introduced in these studies predicts only the tendency to barrelling at the initial instant. The solutions that fall into the other category are based on the concept of sequential limit analysis. Sequential limit analysis is usually used in conjunction with a finite element procedure [11-14]. It is known that faster methods of analysis are needed for some applications [15].

Turning to the cylindrical compression test, friction is responsible for barrelling. This is usually taken into account by assuming a kinematically admissible velocity field that includes a barrel parameter $[5,9,10]$. However, another important effect of friction is that a rigid region must exist in the vicinity of the axis of symmetry. It is well known from experiment [3] and the existence of such a region is predicted by accurate solutions found by the method of characteristics [16]. This region, in turn, leads to the existence of the regime of sticking over a portion of the friction surface. The friction law in terms of stress is not valid where the regime of sticking occurs. Under certain conditions, the regime of sticking occurs over the entire friction surface. In this case, the solution is insensitive to the friction factor. Simple kinematically admissible velocity fields such as those adopted in $[5,9$, 10] do not account for these features of real velocity fields. It is worthy of note that these simple kinematically admissible velocity fields are in general capable of predicting rather accurate upper bounds on the load required to deform the specimen since mathematical properties of the extremum principles of plasticity allow close bounds to be obtained even from crude approximations for the actual stress and velocity fields (it is of course not true for flow pattern) [17]. A general kinematically admissible velocity for axisymmetric forging has been proposed in [18]. This field accounts for the existence of a rigid region and is of the same level of complexity as the fields used in Ebrahimi and Najafizadeh $[5,9,10]$. The objective of the present paper is to combine a particular case of the kinematically admissible velocity field proposed in [18] appropriate for the cylindrical compression test and the concept of sequential limit analysis.

\section{STATEMENT OF THE PROBLEM}

A solid cylinder of radius $R_{0}$ and thickness $2 H_{0}$ is forged between two flat dies moving towards each other with velocity $U$. The current thickness of the cylinder will be denoted by $2 H$. Cylindrical coordinates $(r, \theta, z)$ are taken, with the $z$ - axis taken as the axis of symmetry of the process. Also, the process is symmetric relative to the plane $z=0$. The material of the cylinder is assumed to obey the von Mises yield criterion and its associated flow rule. The tensile yield stress, $\sigma_{0}$, is a material constant. Since $z=0$ is a plane of symmetry for the flow, it is sufficient to consider the region $z \geq 0$. The dies are rough and the friction law is:

$\sigma_{r z}=-m \frac{\sigma_{0}}{\sqrt{3}}$

at $z=H$. Here $\sigma_{r z}$ is the shear stress in the cylindrical coordinates and $m$ is the friction factor, $0 \leq m \leq 1$. Eq. (1) is valid at sliding. At sticking, this equation must be replaced with the condition that the relative velocity between the cylinder and the die vanishes. By symmetry,

$\sigma_{r z}=0$

and $u_{z}=0$ at $z=0$

and $u_{r}=0$ at $r=0$

Moreover,

$u_{z}=-U$ at $z=H$ 
In Eq. (3), Eq. (4) and Eq. (5), $u_{r}$ and $u_{z}$ are the radial and axial velocity, respectively. The outer surface of the cylinder is traction free.

\section{INSTANTANEOUS COMPRESSION}

A general upper bound solution for axisymmetric forging has been proposed in [18]. The corresponding solution for instantaneous compression of a solid cylinder can be derived from this general solution as a special case. In particular, the general structure of the corresponding kinematically admissible velocity field is shown in Fig. 1 where $a c$ is a velocity discontinuity surface. This surface separates plastic and rigid regions. The kinematically admissible velocity field in the plastic region is:

$\frac{u_{r}}{U}=\frac{\rho}{2 h_{0}}+\frac{f(\zeta)}{\rho}, \frac{u_{z}}{U}=-\zeta$

where:

$$
h_{0}=\frac{H_{0}}{R_{0}}, \quad \rho=\frac{r}{R_{0}}, \quad \zeta=\frac{z}{H_{0}}
$$

and $f(\zeta)$ is an arbitrary function of its argument.

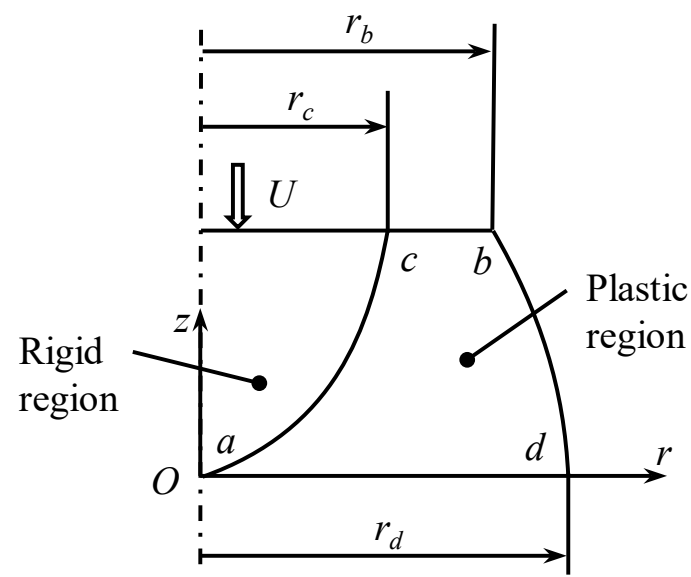

Figure 1 The cylindrical compression test and the general structure of a kinematically admissible velocity field

The power dissipated in the process under consideration is composed of three components: the power dissipated within the plastic region, $W_{V}$; the power dissipated due to plastic shearing at the velocity discontinuity surface $a c, W_{d}$; and the power dissipated by friction at the sliding contact between points $c$ and $b, W_{f}$. All these components have been found in [18]. In particular:

$\frac{W_{V}}{\pi U \sigma_{0} R_{0}^{2}}=\int_{0}^{1} \int_{\eta_{a c}(\zeta)}^{1} \eta^{-1} \sqrt{\eta^{2}+\frac{\eta}{3} g^{2}(\zeta)+\frac{4}{3} h_{0}^{2} f^{2}(\zeta)} \mathrm{d} \eta \mathrm{d} \zeta$

$\frac{W_{d}}{\pi U \sigma_{0} R_{0}^{2}}=\frac{2 \sqrt{2} \sqrt{h_{0}}}{\sqrt{3}}$.

$\int_{0}^{1} \frac{\sqrt{F(\zeta)}}{(1-\zeta)^{3 / 2}}\left[\frac{F(\zeta)}{2(1-\zeta)}+f(\zeta)+\frac{(1-\zeta) f^{2}(\zeta)}{2 F(\zeta)}+h_{0}(1-\zeta)^{2}\right] \mathrm{d} \zeta$

$$
\frac{W_{f}}{\pi U \sigma_{0} R_{0}^{2}}=\frac{m}{\sqrt{3} h_{0}}\left(\frac{2}{3} \eta_{c} \sqrt{\eta_{c}}-\eta_{c}+\frac{1}{3}\right)
$$

In Eq. (8), Eq. (9) and Eq. (10):

$$
\eta=\rho^{2}, \quad g(\zeta)=\frac{\mathrm{d} f}{\mathrm{~d} \zeta}, \quad F(\zeta)=\int_{1}^{\zeta} f(\chi) \mathrm{d} \chi
$$

Moreover:

$$
\eta=\eta_{a c}(\zeta)=\frac{2 h_{0} F(\zeta)}{1-\zeta}
$$

is the equation of the velocity discontinuity surface ac and $\eta_{c}$ is the value of $\eta$ at point $c$. Eq. (8), Eq. (9) and Eq. (10) are valid if point $c$ is closer to the axis of symmetry than point $b$. This condition will be verified a posteriori. Let $P_{u}$ be an upper bound on the force required to deform the cylinder and $p_{u}$ is its dimensionless representation. Then:

$$
p_{u}=\frac{P_{u}}{\pi \sigma_{0} R_{0}^{2}}=\frac{W_{V}+W_{d}+W_{f}}{\pi U \sigma_{0} R_{0}^{2}}
$$

Substituting Eq. (8), Eq. (9) and Eq. (10) into Eq. (13) shows that $p_{u}$ depends on $f(\zeta)$. It is advantageous to choose this function such that some boundary conditions not involved in the upper bound theorem are satisfied. In particular, it follows from Eq. (2) and the associated flow rule that $\xi_{r z}=0$ at $\zeta=0$ where $\zeta_{r z}=0 \xi_{r z}$ is the shear strain rate in the cylindrical coordinate system. Since $2 \xi_{r z}=\partial u_{r} / \partial z+\partial u_{z} / \partial r$ and $u_{z}$ is independent of $r$ as follows from Eq. (6), the boundary condition Eq. (2) requires that:

$$
\frac{\partial u_{r}}{\partial \zeta}=0
$$

at $\zeta=0$. Moreover, it is known [19] that

$\xi_{r z}=O\left(\frac{1}{\sqrt{\zeta}}\right)$

as $\zeta \rightarrow 1$ if $m=1$ in Eq. (1). If $m \neq 1$ then Eq. (15) is not valid. Nevertheless, velocity fields satisfying Eq. (15) are kinematically admissible at any value of $m$. Moreover, numerical results reported in [20] demonstrated that the use of Eq. (15) led to a better upper bound prediction of the extrusion pressure for a wide range of $m$, compared with other kinematically admissible velocity fields of the same level of complexity. Therefore, it makes sense to use Eq. (15) if $\mathrm{m}$ is large enough. One of the simplest functions satisfying Eq. (14) and Eq. (15) is:

$$
f(\zeta)=-\frac{\eta_{c}}{2 h_{0}}-\beta \sqrt{1-\zeta^{2}}
$$


Here $\beta$ is constant. Eq. (11) and Eq. (16) combine to give:

$$
\begin{aligned}
& g(\zeta)=\frac{\beta \zeta}{\sqrt{1-\zeta^{2}}}, \\
& F(\zeta)=-\frac{\eta_{c} \zeta+\beta h_{0} \zeta \sqrt{1-\zeta^{2}}+\beta h_{0} \arcsin \zeta}{2 h_{0}}
\end{aligned}
$$

Since $\eta=\eta_{c}$ at $\zeta=1$, it follows from Eq. (12) and Eq. (17) that $\beta=-2 \eta_{c} /\left(\pi h_{0}\right)$. Then, Eq. (16) and Eq. (17) become:

$$
\begin{aligned}
& f(\zeta)=-\frac{\eta_{c}}{2 h_{0}}\left(1-\frac{4}{\pi} \sqrt{1-\zeta^{2}}\right), \\
& g(\zeta)=-\frac{2 \eta_{c} \zeta}{\pi h_{0} \sqrt{1-\zeta^{2}}}, \\
& F(\zeta)=\frac{\eta_{c}}{2 h_{0}}\left[\frac{2}{\pi}\left(\arcsin \zeta+\zeta \sqrt{1-\zeta^{2}}\right)-\zeta\right]
\end{aligned}
$$

Substituting Eq. (18) into Eq. (8), Eq. (9), Eq. (10), and Eq. (12) and, then, the resulting expressions into Eq. (13) supplies $p_{u}$ as a function of $\eta_{c}$. Minimizing this function with respect to $\eta_{c}$ provides the best upper bound on $p_{u}$ based on the kinematically admissible velocity field chosen.

Numerical minimization of the right hand side of Eq. (13) has shown that the solution predicts the existence of the rigid region only if $m>0,7$ (approximately). Therefore, further analysis is restricted to the range $0,7 \leq m \leq 1$. It has been also confirmed that the condition $\eta_{c}<1$ is satisfied (i.e. the general structure of the kinematically admissible velocity field shown in Fig. 1 is correct).

\section{CONTINUED COMPRESSION}

The shape of the cylinder after any amount of compression is schematically illustrated in Fig. 1. No velocity boundary conditions are prescribed on the traction free surface $b d$. The velocity field Eq. (6) satisfies the boundary condition Eq. (5) if $\zeta$ is defined as

$\zeta=\frac{z}{H}$

Therefore, the velocity field Eq. (6) is kinematically admissible independently of the shape of $b d$ if $h_{0}$ is replaced with $h$ where $h=H / R_{0}$. Then, $h_{0}$ should be replaced with $h$ in Eq. (8), Eq. (9) and Eq. (10). At the initial instant $h=h_{0}$ and the definitions for $\zeta$ given in Eq. (7) and Eq. (19) coincide. The integrand in Eq. (8) is independent of the shape of $b d$ but one of the limits of integration is. Therefore, Eq. (8) becomes:

$$
\frac{W_{V}}{\pi U \sigma_{0} R_{0}^{2}}=\int_{0}^{1} \int_{\eta_{a c}(\zeta)}^{\eta_{b d}(\zeta)} \eta^{-1} \sqrt{\eta^{2}+\frac{\eta}{3} g^{2}(\zeta)+\frac{4}{3} h^{2} f^{2}(\zeta)} \mathrm{d} \eta \mathrm{d} \zeta
$$

where $\eta=\eta_{b d}(\zeta)$ is the equation of the traction free surface bd. The integrand and the limits of integration in Eq. (9) are independent of the shape of $b d$. Therefore, this equation becomes:

$$
\begin{aligned}
& \frac{W_{d}}{\pi U \sigma_{0} R_{0}^{2}}=\frac{2 \sqrt{2} \sqrt{h}}{\sqrt{3}} \\
& \int_{0}^{1} \frac{\sqrt{F(\zeta)}}{(1-\zeta)^{3 / 2}}\left[\frac{F(\zeta)}{2(1-\zeta)}+f(\zeta)+\frac{(1-\zeta) f^{2}(\zeta)}{2 F(\zeta)}+h(1-\zeta)^{2}\right] \mathrm{d} \zeta
\end{aligned}
$$

The power dissipated by friction at the sliding contact between points $c$ and $b$ is found from the equation

$$
W_{f}=\frac{2 \pi m \sigma_{0}}{\sqrt{3}} \int_{r_{c}}^{r_{b}}\left|u_{r}\right| r \mathrm{~d} r
$$

where $r_{b}$ is the radial coordinate of point $b$ and $r_{c}$ is the radial coordinate of point $c$. In Eq. (22), the radial velocity is understood to be calculated at $\zeta=1$. Using Eq. (7) and Eq. (11). Eq. (22) can be transformed to:

$$
W_{f}=\frac{\pi m \sigma_{0} R_{0}^{2}}{\sqrt{3}} \int_{\eta_{c}}^{\eta_{b}}\left|u_{r}\right| \mathrm{d} \eta
$$

where $\eta_{c}=\left(r_{c} / \mathrm{R}_{0}\right)^{2}$ and $\eta_{b}=\left(r_{b} / \mathrm{R}_{0}\right)^{2}=\eta_{b d}(1)$. It has been shown in [18] that $\eta_{c}=-2 h f$ (1) independently of the function $f(\zeta)$. Therefore, using Eq. (6) and Eq. (11). Eq. (23) can be rewritten as:

$$
\begin{aligned}
& \frac{W_{f}}{\pi U \sigma_{0} R_{0}^{2}}=\frac{m}{2 h \sqrt{3}} \int_{\eta_{c}}^{\eta_{b}}\left(\sqrt{\eta}-\frac{\eta_{c}}{\sqrt{\eta}}\right) \mathrm{d} \eta= \\
& =\frac{m}{3 \sqrt{3} h}\left(\eta_{b}^{3 / 2}-3 \eta_{c} \sqrt{\eta_{b}}+2 \eta_{c}^{3 / 2}\right)
\end{aligned}
$$

This equation replaces Eq. (10). It is evident that Eq. (10) and Eq. (24) coincide at the initial instant when $\eta_{b}=$ 1. Substituting Eq. (18) in which $h_{0}$ is replaced with $h$ into Eq. (20), Eq. (21), Eq. (24), and Eq. (12) and, then, the resulting expressions into Eq. (13) supplies $p_{u}$ as a function of $\eta_{c}$. In Eq. (12) and Eq. (18) it is necessary to replace $h_{0}$ with $h$. Minimizing $p_{u}$ with respect to $\eta_{c}$ provides the best upper bound on $p_{u}$ based on the kinematically admissible velocity field chosen. However, in contrast to the solution given in the previous section, the shape of the traction free surface $b d$ is unknown and should be found from the solution. By definition:

$U \frac{\mathrm{d} z}{\mathrm{~d} H}=\frac{U}{R_{0}} \frac{\mathrm{d} z}{\mathrm{~d} h}=-u_{z}, \quad U \frac{\mathrm{d} r}{\mathrm{~d} H}=\frac{U}{R_{0}} \frac{\mathrm{d} r}{\mathrm{~d} h}=-u_{r}$

Substituting Eq. (6) into Eq. (25) and using Eq. (7) to eliminate $r$, Eq. (11) to eliminate $\rho$ and Eq. (19) to eliminate $\zeta$ in the second equation in Eq. (6) yields:

$$
\frac{\mathrm{d} \eta}{\mathrm{d} h}=-\frac{\eta}{h}-2 f(\zeta, h), \quad \frac{\mathrm{d} z}{\mathrm{~d} h}=\frac{z}{h}
$$


The notation $f(\zeta, h)$ emphasizes that, in contrast to the solution given in the previous section, the function $f$ depends on both $\zeta$ and $h$. The second equation in Eq. (26) can be immediately integrated to give:

$$
\frac{Z}{h_{0}}=\frac{z}{h}
$$

where $Z$ is the Lagrangian coordinate such that $Z=z$ at the initial instant. It is seen from Eq. (19) and Eq. (27) that $\mathrm{Z} / H_{0}=\zeta$ and, therefore, $\zeta$ is a Lagrangian coordinate as well. Then, the first equation in Eq. (26) can be integrated. Let $Y$ be the Lagrangian coordinate such that $Y=\rho$ at the initial instant. The solution of the first equation in Eq. (26) satisfying the condition $\gamma=\rho$ at $h=h_{0}\left(\right.$ or $\left.H=H_{0}\right)$ is:

$\eta=\frac{1}{h}\left(r^{2} h_{0}-2 \int_{h_{0}}^{h} t f(\zeta, t) \mathrm{d} t\right)$

At the initial instant the equation of the traction free surface bd is $Y=1$. Therefore, it follows from Eq. (28) that the equation of this surface after any amount of deformation is:

$\eta=\eta_{b d}(\zeta, h)=\frac{1}{h}\left(h_{0}-2 \int_{h_{0}}^{h} t f(\zeta, t) \mathrm{d} t\right)$

Eq. (18) in which $h_{0}$ should be replaced with h may be used to specify the function $f$. In particular:

$$
\begin{aligned}
& f(\zeta, h)=-\frac{\eta_{c}}{2 h}\left(1-\frac{4}{\pi} \sqrt{1-\zeta^{2}}\right), \\
& g(\zeta, h)=-\frac{2 \eta_{c} \zeta}{\pi h \sqrt{1-\zeta^{2}}}, \\
& F(\zeta, h)=\frac{\eta_{c}}{2 h}\left(\frac{2}{\pi}\left(\arcsin \zeta+\zeta \sqrt{1-\zeta^{2}}\right)-\zeta\right)
\end{aligned}
$$

It is worthy of note that $\eta_{c}$ depends on $h$. Substituting Eq. (30) into Eq. (29) yields:

$$
\begin{aligned}
& \eta_{b d}(\zeta, h)=\frac{h_{0}}{h}+\frac{1}{h}\left(1-\frac{4 \sqrt{1-\zeta^{2}}}{\pi}\right) I(h), \\
& I(h)=\int_{h_{0}}^{h} \eta_{c}(t) \mathrm{d} t
\end{aligned}
$$

Eq. (12) in which $h_{0}$ should be replaced with $h$, Eq. (20), Eq. (21), Eq. (24), Eq. (30) and Eq. (31) allow the right hand side of Eq. (13) to be found at any value of $h$. It is however necessary to develop a procedure for evaluating the integral in Eq. (31). A simple predictor-corrector method is adopted. Let $\eta_{c}^{(i)}$ be the value of $\eta_{c}$ and $I^{(i)}$ be the value of I at $h=h_{\mathrm{i}}$. It is worthy of note that the value of $\eta_{c}$ at $h=h_{0}$ is found using the solution given in the previous section. Therefore, this value is supposed to be known. It is also obvious that $I=0$ at $h=h_{0}$. The predictor equation to get $I^{(i+l)}$ is

$$
\tilde{I}^{(i+1)}=I^{(i)}+\eta_{c}^{(i)} \Delta h
$$

Here $\Delta h$ is an increment of $h$. Using $\tilde{I}^{(i+1)}$ the corresponding shape of the traction free surface $b d$ is determined from Eq. (31) and then an initial guess value of $\tilde{\eta}_{c}^{(i+1)}$ is found by minimizing $p_{u}$ given by Eq. (13). The corrector equation to get $I^{(i+I)}$ is:

$I^{(i+1)}=I^{(i)}+\tilde{\eta}_{c}^{(i)} \Delta h$

Using $I^{(i+I)}$ the final shape of the traction free surface $b d$ at $h=h^{(i+I)}$ is determined from Eq. (31) and then the value of $\eta_{c}^{(i+1)}$ is found by minimizing $p_{u}$ given by Eq. (13).

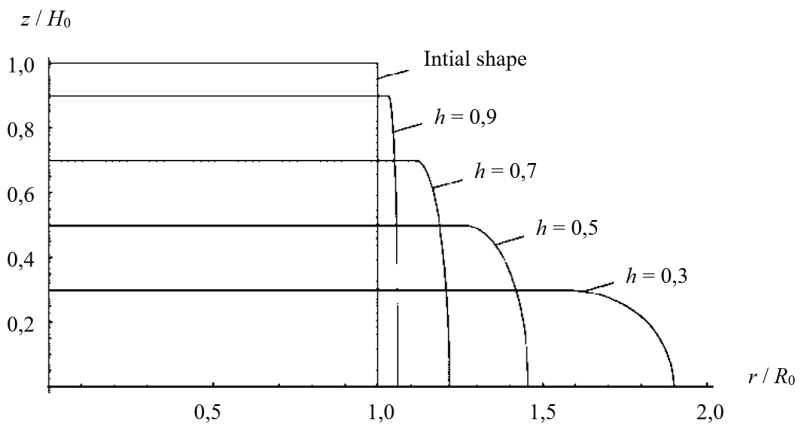

Figure 2 Contour of the specimen in the $(r, z)$ - plane at $m=0,8$ and several values of $h$

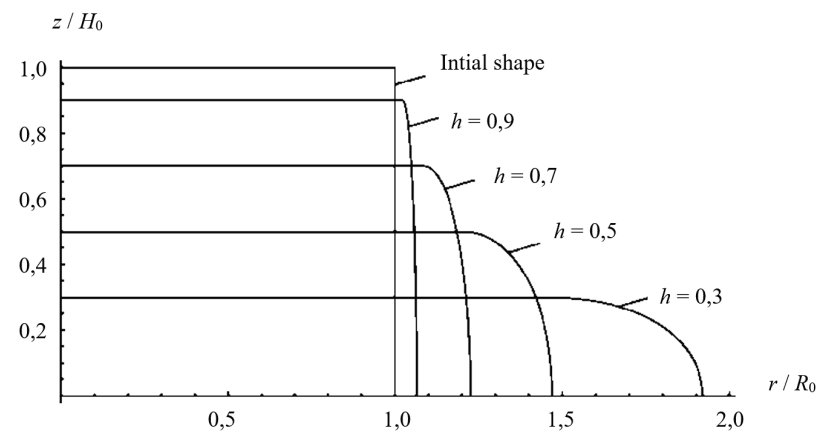

Figure 3 Contour of the specimen in the $(r, z)$ - plane at $m=1$ and several values of $h$

As an illustration of the method developed the evolution of surface $b d$ has been found at $m=0,8$ and $m=$ 1 . In both cases $h_{0}=1$. The contour of the cylinder at several values of $h$ is shown in Fig. 2 and Fig. 3 at $m=0,8$ and $m=1$, respectively. The variation of $p_{u}$ with $h$ is depicted in Fig. 4 at several values of $m$ in the range $0,7 \leq m \leq 1$. However, the effect of $m$ on $p_{u}$ is so small that the difference between the curves corresponding to different values of $\mathrm{m}$ is invisible. The values of $r_{b}$ and $r_{d}$ are important for methods used to determine the friction factor [8].

Therefore, the variation of $\rho_{b}=r_{b} / R_{0}$ and $\rho_{d}=r_{d} / R_{0}$ with $h$ is shown in Fig. 5 at several values of $m$. The broken curves correspond to $\rho_{d}$. It is seen from this figure that the effect of $\mathrm{m}$ on this parameter is very week. The finite 
element solution presented in [8] demonstrates the same tendency. The parameter $\rho_{b}$ is influenced by the friction factor.

This is also in qualitative agreement with the aforementioned finite element solution.

\section{CONCLUSIONS}

An analytical investigation of the cylindrical compression test has been conducted. The method developed combines rather a simple kinematically admissible velocity field proposed in [18] and the concept of sequential limit analysis. This simplicity of the kinematically admissible velocity field comes at cost: as compared to general sequential limit analyses boundary value problems that belong to a certain class can only be solved by the method developed. Nevertheless, this class is rather large. For example, the boundary value problems formulated in $[6,21,22]$ can be treated without any change in the general procedure. The ring compression test can be treated by combining the general kinematically admissible velocity field [18] and the general procedure developed in the present paper. Some modifications of the general kinematically admissible velocity field are required to solve the boundary value problem presented in [23] and to treat upsetting of hollow and solid cylinders with rotating tool. The latter process is important for applications [2427]. In addition, the method proposed can be extended to strain and work hardening materials in the same manner as this material property is incorporated in conventional sequential limit analyses [11, 12, 28, 29].

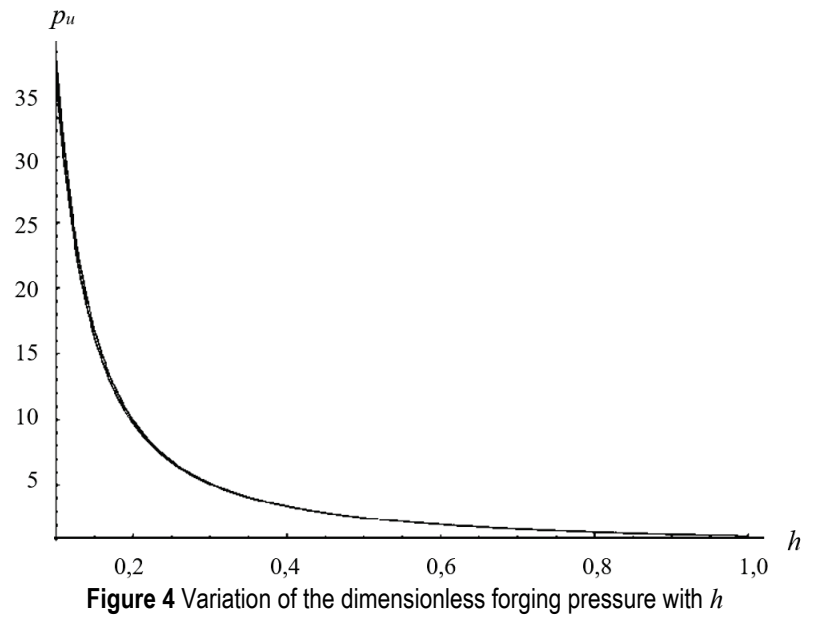

The solution found in the present paper is appropriate for higher values of $m$. In particular, the solution predicts no rigid region at $m>0,7$ (approximately). This is a consequence of the assumption made in [18] that the rigid region penetrates the thickness of the specimen. The solution for the range $1 \geq m \geq 0,7$ is illustrated in Fig. 4 and Fig. 5. It is seen from Fig. 4 that the load required to deform the specimen is not sensitive to the friction factor. This is an effect of the rigid region. In particular, the power is not dissipated where the regime of sticking occurs. The regime of sliding occurs in the range $\rho_{b} \leq \rho \leq \rho_{d}$. It is seen from Fig. 5 that the area of sliding contact is rather small. It is also seen from this figure that the parameter $\rho_{d}$ is not sensitive to the friction factor. Therefore, the only parameter that is sensitive to the friction factor in the range $1 \geq m \geq 0,7$ is $\rho_{b}$. The same tendency in the dependence of $\rho_{b}$ and $\rho_{d}$ on $m$ has been found in [8] by means of the finite element method.

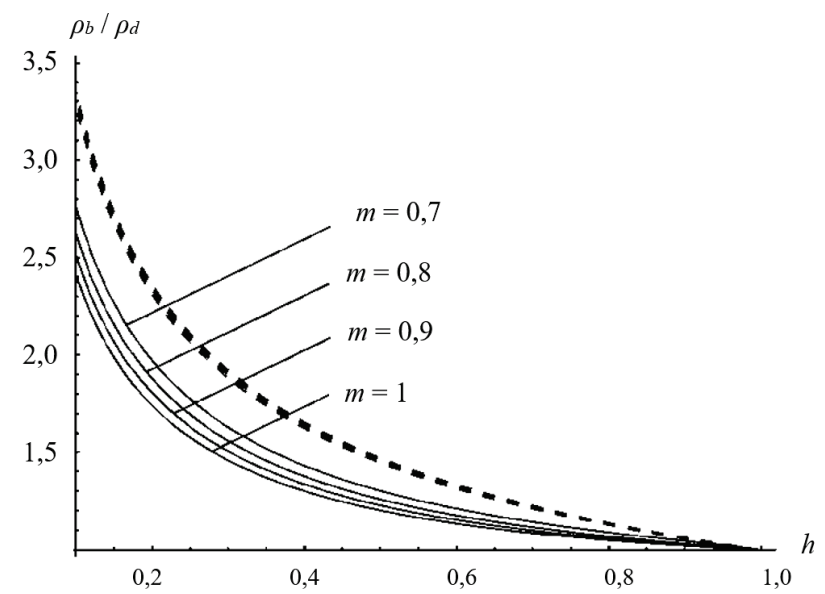

Figure 5 Variation of $\rho_{b}$ and $\rho_{d}$ with $h$ at several values of $m$ (the solid curves correspond to $\rho_{b}$ and the broken curves to $\rho_{d}$ )

\section{Acknowledgements}

This work was carried out within the framework of a joint project supported by grants RFBR-18-51-76001 (Russia) and BULKSURFACE-359 (Ministry of education, science and technological development, Serbia).

\section{REFERENCES}

[1] Hsu, T. C. \& Young, A. J. (1967). Plastic deformation in the compression test of pure copper. The Journal of Strain Analysis for Engineering, 2(2), 159-170. https://doi.org/10.1243/03093247V022159

[2] Cohen, R. E. \& Durham, D. R. (1990). Microstructure as a criterion for the selection of hot working process parameters for plain medium carbon steel. Journal of Engineering Materials and Technology, 112(1), 90-94. https://doi.org/10.1115/1.2903192

[3] Kuhn, H. A. (2003). Cold Upset Testing. In: G. E. Dieter, H. A. Kuhn, \& S. L. Semiatin (Eds.), Handbook of workability and process design (pp. 57-60). ASM International.

[4] Narayanan, R. G., Gopal, M., \& Rajadurai, A. (2008). Influence of friction in simple upsetting and prediction of hardness distribution in a cold forged product. Journal of Testing and Evaluation, 36(4), Paper JTE101443. https://doi.org/10.1520/JTE101443

[5] Ebrahimi, R. \& Najafizadeh, A. (2004). A new method for evaluation of friction in bulk metal forming. Journal of Materials Processing Technology, 152(2), 136-143. https://doi.org/10.1016/j.jmatprotec.2004.03.029

[6] Malayappan S. \& Esakkimuthu, G. (2006). Barrelling of aluminium solid cylinders during cold upsetting with differential conditions at the faces. The InternationalJournal of Advanced Manufacturing Technology, 29(41), 41-48. https://doi.org/10.1007/s00170-005-2503-6

[7] Cetinarslan, C. S. (2007). Effect of aspect ratio on barreling contour and variation of total surface area during upsetting of cylindrical specimen. Materials \& Design, 28, 1907-1913. https://doi.org/10.1016/j.matdes.2006.03.027

[8] Li, Y. P., Onodera, E., \& Chiba, A. (2010). Evaluation of friction coefficient by simulation in bulk metal forming process. Metallurgical and Materials Transactions A, 41, 224-232. https://doi.org/10.1007/s11661-009-0066-0 
[9] Liu, J. Y. (1971). Upper - bound solutions of some axisymmetric cold forging problems.Trans. ASME Journal of Engineering for Industry, 93(4), 1134-1144. https://doi.org/10.1115/1.3428053

[10] Solhjoo, S. (2010). A note on "Barrel Compression Test": a method for evaluation of friction. Computational Materials Science, 49(2), 435-438. https://doi.org/10.1016/j.commatsci.2010.04.047

[11] Huh, H. \& Lee, C, H. (1993). Eulerian finite-element modeling of the extrusion process for work-hardening materials with the extended concept of limit analysis. Journal of Materials Processing Technology, 38(1-2), 51-62. https://doi.org/10.1016/0924-0136(93)90185-9

[12] Hwan, C. L. (1997). Plane strain extrusion by sequential limit analysis. International Journal of Mechanical Sciences, 39(7), 807-817. https://doi.org/10.1016/S0020-7403(96)00089-6

[13] Chang, C. C. \& Bramley, A. N. (2000). Forging preform design using a reverse simulation approach with the upper bound finite element procedure. Proceedings of the Institution of Mechanical Engineers, Part C: Journal of Mechanical Engineering Science, 214(1), 127-136. https://doi.org/10.1243/0954406001522868

[14] Mirnia, M. J., Dariani, B. M., Vanhove, H., \& Duflou, J. R. (2014). An investigation into thickness distribution in single point incremental forming using sequential limit analysis. International Journal of Material Forming, 7(4), 469-477. https://doi.org/10.1007/s12289-013-1143-x

[15] Cawthorn, C. J., Loukaides, E. G., \& Allwood, J. M. (2014). Comparison of analytical models for sheet rolling. Procedia Engineering, 81, 2451-2456. https://doi.org/10.1016/j.proeng.2014.10.349

[16] Druyanov, B. \& Nepershin, R. (1994). Problems of Technological Plasticity. Amsterdam, Elsevier.

[17] Hill, R. (1956). New horizons in the mechanics of solids. Journal of the Mechanics and Physics of Solids, 5(1), 66-74. https://doi.org/10.1016/0022-5096(56)90010-2

[18] Alexandrov, S., Lyamina, E., \& Jeng, J. R. (2017). A general kinematically admissible velocity field for axisymmetric forging and its application to hollow disk forging. The International Journal of Advanced Manufacturing Technology, 88(9-12), 3113-3122. https://doi.org/10.1007/s00170-016-9018-1

[19] Alexandrov, S., \& Richmond, O. (2001). Singular plastic flow fields near surfaces of maximum friction stress. International Journal of Non-Linear Mechanics, 36(1), 1-11. https://doi.org/10.1016/S0020-7462(99)00075-X

[20] Alexandrov, S., Mishuris, G., Mishuris, W., \& Sliwa, R. E. (2001). On the dead zone formation and limit analysis in axially symmetric extrusion. International Journal of Mechanical Sciences, 43(2), 367-379. https://doi.org/10.1016/S0020-7403(00)00016-3

[21] Thaheer, A. S. A. \& Narayanasamy, R. (2008). Comparison of barreling in lubricated truncated cone billets during cold upset forging of various metals. Materials \& Design, 29(5), 1027-1035. https://doi.org/10.1016/j.matdes.2006.05.026

[22] Zhbankov, I. G., Markov, O. E., \& Perig, A. V. (2014). Rational parameters of profiled workpieces for an upsetting process. The International Journal of Advanced Manufacturing Technology, 72(5-8), 865-872. https://doi.org/10.1007/s00170-014-5727-5

[23] Manisekar, K., Narayanasamy, R., \& Malayappan, S. (2006). Effect of friction on barrelling in square billets of aluminium during cold upset forging. Materials \& Design, 27(2), 147155. https://doi.org/10.1016/j.matdes.2004.09.019

[24] Kemin, X., Zhen, W., \& Yan, L. (1997). FEM analysis of cylinder twist-compression deformation regularity. Journal of Materials Processing Technology, 69(1-3), 148-151.
https://doi.org/10.1016/S0924-0136(97)00008-3

[25] Park, J. H., Kim, Y. H., \& Jin, Y. E. (2001). Experimental investigation of the forming parameters of the rotational upset forging process. Journal of Materials Processing Technology, 111(1-3), 103-106. https://doi.org/10.1016/S0924-0136(01)00490-3

[26] Huang, M. N., \& Tzou, G. Y. (2002). Study on compression forming of a rotating disk considering hybrid friction. Journal of Materials Processing Technology, 125-126, 421426. https://doi.org/10.1016/S0924-0136(02)00293-5

[27] Ma, X., Barnett, M. R., \& Kim, Y. H. (2003). Experimental and theoretical investigation of compression of a cylinder using a rotating platen. International Journal of Mechanical Sciences, 45(10), 1717-1737. https://doi.org/10.1016/j.jimecsci.2003.12.003

[28] Hwan, C. L. (1997). An upper bound finite element procedure for solving large plain strain deformation. International Journal for Numerical Methods in Engineering, 40(10), 1909-1922. https://doi.org/10.1002/(SICl)10970207(19970530)40:10<1909::AID-NME149>3.0.CO;2-0

[29] Leu, S-Y. (2005). Convergence analysis and validation of sequential limit analysis of plane-strain problems of the von Mises model with non-linear isotropic hardening. International Journal for Numerical Methods in Engineering, 64(3), 322-334. https://doi.org/10.1002/nme.1367

\section{Contact information}

\section{Sergei ALEXANDROV, PhD}

Division of Computational Mathematics and Engineering, Institute for Computational Science, Ton Duc Thang University, 19 Nguyen Huu Tho Street, Ho Chi Minh City 700000, Vietnam Faculty of Civil Engineering, Ton Duc Thang University, 19 Nguyen Huu Tho Street, Ho Chi Minh City 700000, Vietnam E-mail: sergeialexandrov@tdtu.edu.vn

Dmitry LISOVENKO, PhD, Associate Professor Ishlinsky Institute for Problems in Mechanics RAS, 101-1 Prospect Vernadskogo, 119526 Moscow, Russia E-mail: lisovenk@ipmnet.ru

Marko VILOTIC, PhD, Professor Assistant

(Corresponding author)

Faculty of Technical Sciences, University of Novi Sad,

Trg Dositeja Obradovica 6, 21000 Novi Sad, Serbia

E-mail: markovil@uns.ac.rs 